\title{
Targeted delivery of in situ PCR-amplified Sleeping Beauty transposon genes to cancer cells with lipid-based nanoparticle-like protocells
}

Kun $\mathrm{Ma}^{\mathrm{a}, \mathrm{b}^{*}}$, Duo Fu${ }^{\mathrm{a}}$, Dongli $\mathrm{Yu}^{\mathrm{a}}$, Changhao Cui ${ }^{\mathrm{a}}$, Li Wang ${ }^{\mathrm{a}}$, Zhaoming Guo ${ }^{\mathrm{a}}$, Chuanbin $\mathrm{Mao}^{\mathrm{b}, \mathrm{c}^{* *}}$

a. School of Life Science and Medicine, Dalian University of Technology, Panjin 124221, China

b. Department of Chemistry and Biochemistry, Stephenson Life Sciences Research

Center, University of Oklahoma, OK 73019, USA

c. School of Materials Science and Engineering, Zhejiang University, Hangzhou, Zhejiang 310027, China

*Corresponding author: School of Life Science and Medicine, Dalian University of Technology Panjin Campus, Road of DUT, New District of LiaoDong Gulf, PanJin, Liaoning province 124221, China

**Co-corresponding author: Department of Chemistry and Biochemistry, University of Oklahoma, Stephenson Life Sciences Research Center, 101 Stephenson Parkway, Norman, OK 73019, USA

E-mail address: makunonline@163.com (Kun Ma), cbmao@ou.edu (Chuanbin Mao)

\begin{abstract}
A Sleeping Beauty (SB) transposon system is made of a transposon plasmid (containing gene encoding a desired functional or therapeutic protein) and a transposase plasmid (encoding an enzyme capable of cutting and pasting the gene into the host cell genome). It is a kind of natural, nonviral gene delivery vehicle, which can achieve efficient genomic insertion, providing long-term transgenic expression. However, before the SB transposon system could play a role in promoting gene expression, they have to be delivered efficiently first across cell membrane and then into cell nuclei. Towards this end, we used a nanoparticle-like lipid-based protocell, a closed bilayer of the neutral lipids with the DNA encapsulated inside, to deliver the SB transposon system to cancer cells. The SB transposon system was amplified in situ inside the protocells by a polymerase chain reaction (PCR) process, realizing more efficient loading and delivery of the target gene. To reach a high transfection efficiency, we introduced two targeting moieties, folic acid (FA) as a cancer cell-targeting motif and Dexamethasone (DEX) as a nuclear localization signaling molecule, into the protocells. As a result, the FA enabled the modified targeting protocells to deliver the DNA into the cancer cells with an
\end{abstract}


increased efficiency and the DEX promoted the DNA to translocate to cell nuclei, eventually leading to the increased chromosomes insertion efficiency of the SB transposon. In vivo study strongly suggested that the transfection efficiency of FAmodified protocells in the tumor tissue was much higher than that in other tissues, which was consistent with the in vitro results. Our studies implied that with the targeting ligand modification, the protocells could be utilized as an efficient targeting gene carrier. Since the protocells were made of neutral lipids without cationic charges, the cytotoxicity of protocells was significantly lower than that of traditional cationic gene carrier such as cationic liposomes and polyethylenimine, enabling the protocells to be employed in a wider dosage range in gene therapy. Our work shows that the protocells are a promising gene carrier for future clinical applications.

Key Words: Sleeping Beauty transposon; Protocells; Non-viral gene carrier; Targeted delivery; Cancer cells

\section{Introduction}

Gene therapy holds promise for treating a variety of diseases including cancer. However, its clinical application is limited by the deficiency of safe and efficient methods for gene delivery. At least three biological barriers, including the cellular membrane, the nuclear membrane, and chromosomal integrity, should be penetrated by the gene delivery systems to achieve high gene transfection efficiency. At present, two broad classes of vectors, viral and nonviral, are employed to deliver genes to mammalian chromosomes [1]. Viral vectors can achieve a high gene transfer efficiency. However, viral vectors may insert the recombinant virus in chromosomes, resulting in carcinomatous mutation. Furthermore, they are inherently immunogenic with high immune reactions [2]. On the other hand, non-viral vectors show advantages such as improved safety, greater flexibility and more controllable manufacturing, although their low transfection efficiency compared to viral vectors hampers the clinical application [3].

Sleeping Beauty (SB) transposon system is a kind of natural, nonviral gene delivery vehicles capable of achieving efficient genomic insertion [4, 5]. It is a plasmid-based system constituting a transposon and a transposase that can insert a specific fragment of 
DNA into genomes of cells in a cut-and-paste fashion. Hence, it can promote long-term expression of the interest gene [6]. However, in order for it to be functional in promoting gene expression, it has to be delivered efficiently first across cell membrane and then into cell nuclei [7].

Traditional non-viral gene carriers such as cationic DOTAP liposomes contain strong cationic charges to efficiently load anionic genetic materials, such as a plasmid, antisense oligodeoxynucleotide, or siRNA [8]. However, the quantity of genetic materials that were loaded by cationic carriers is limited, depending on the positive charge of the carrier [9]. Furthermore, the cationic character of these carriers will lead to destabilization of plasma membranes, causing severe cytotoxicity. These features impede the clinical application of cationic non-viral gene carriers critically.

Protocells, a nano-bioreactor formed by neutral lipid bilayer (mimicking cell membranes) and showing a morphological similarity with liposomes, have been employed as an essential tool for understanding the origin of life [10]. It has been reported that various biochemical reactions, such as genome replication and protein synthesis, can occur inside the protocells without damaging them [11-13]. It has been reported that DNA amplification could take place inside the lipid-based nanoparticles via PCR technique [14] [15]. Hence, we conjecture that DNA amplification can also occur inside the protocells. Namely, initially loaded DNA molecules can be further amplified by PCR in situ inside the protocells, allowing us to load a large quantity of DNA in gene delivery. The traditional cationic liposomes condense DNA through electrostatic interaction, which limits the quantity of DNA that can be delivered. Therefore, we propose to use protocells to deliver the DNA, which is amplified in situ inside and protected by the protocells. Although the protocells have low in vivo toxicity due to their neutral charge compared to cationic liposomes, their neutral charge reduces their capability to transfer DNA into cells, limiting their transfection efficiency and thus the eventual application in gene therapy.

Therefore, to achieve high transfection efficiency of the protocells for cancer gene therapy, in this work we propose to use protocells, which are modified to bear cancer cell-targeting and nuclei-targeting capability, to deliver the aforementioned SB transposon systems into cancer cells (Fig. 1). Firstly, the SB transposon system was 

for efficient delivery. Then, the protocells were modified with two active targeting moieties. One is folic acid (FA) containing polyethylene glycol distearoylphosphatidylethanolamine (FA-PEG-DSPE), which enables the FA motif to be exposed on the surface of the protocells for increasing the cellular uptake of transposons gene [16]. Another is dexamethasone (DEX), which was embedded in the hydrophobic bilayer and employed as a nuclear localization signaling molecule for improving the nuclear translocation of transposons to favor the insertion of transgenes into the host genome [17]. Our studies showed that protocells modified with cancer cell targeting motifs deliver the SB transposon system, which was amplified in situ by PCR and well protected by the neutral lipid bilayers, to cancer cell nuclei, followed by sustained expression of the target gene with an improved efficiency compared to traditional cationic liposomes.

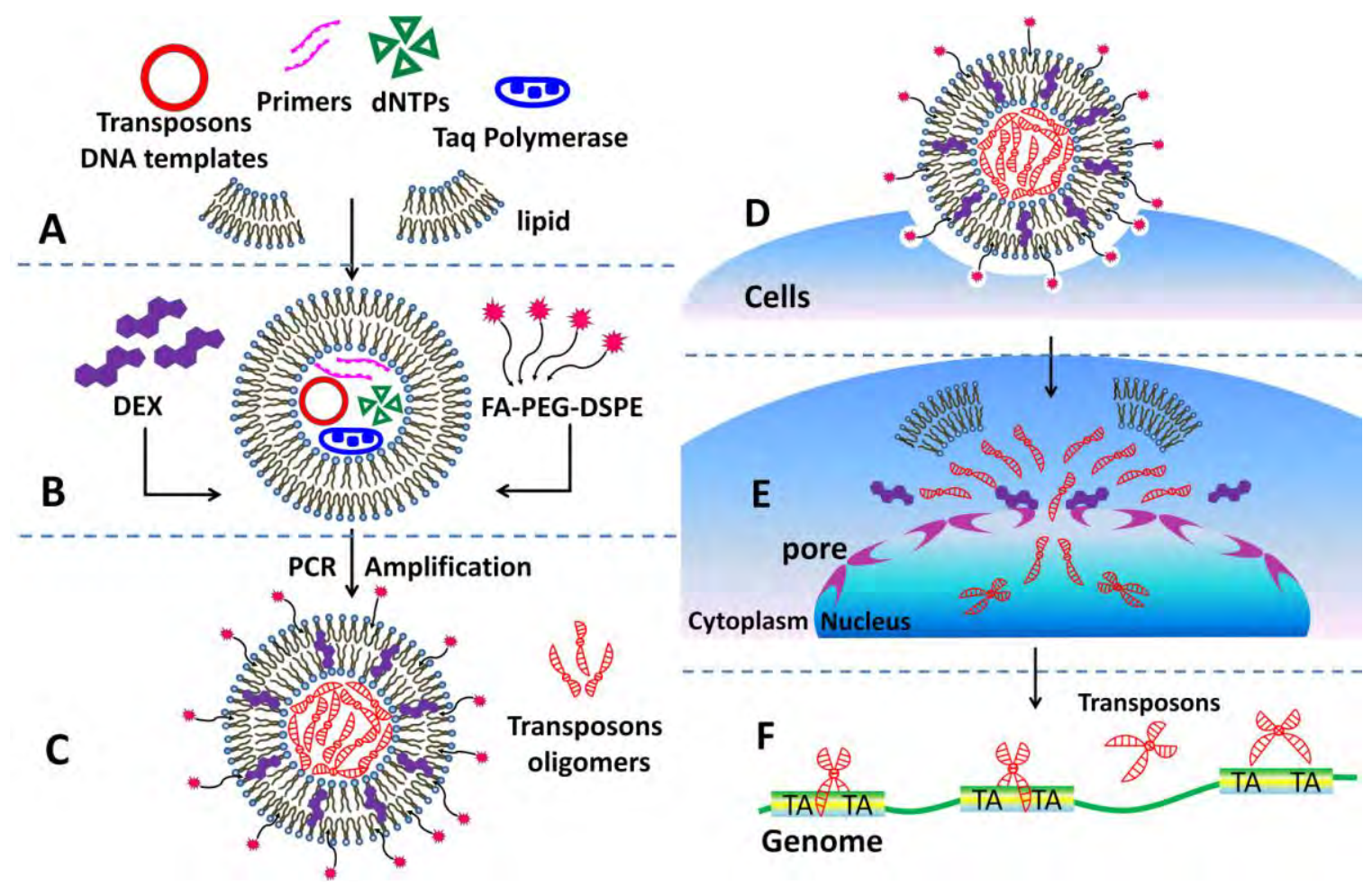

Fig. 1. Schematic illustration of preparation of FA-protocells and the targeted delivery process of FA-protocells in Hela cells. A: PCR components (DNA template, primers, dNTPs and polymerase) are encapsulated into neutral lipid-based protocells. The DNA template is a transposon encoding EGFP ( $\mathrm{pT}_{2} \mathrm{EGFP}$ ) or a plasmid encoding transposase 
(SB11). B: Protocells are modified with FA-PEG $2000-\mathrm{DSPE}$ (for target cancer cells) and DEX (for targeting cell nuclei) to form FA-protocells. C: After PCR amplification, a high concentration of transposon and transposase genes is manufactured inside the protocells. D: FA-protocells are uptaken into cells efficiently by receptor-mediated endocytosis. E: Inside the cells, DEX can dilate the nuclear pore to facilitate the uptake of transposons into the nucleus. F: Inside the cell nucleus, an interest gene was inserted into a genomic location containing TA dinucleotides by transposons with a "cut-and-paste" mechanism.

\section{Materials and methods}

\subsection{Materials}

1,2-Dipalmitoyl-sn-glycero-3-phosphocholine（DPPC), 1,2-dioleoyl-sn-glycero-3phosphoethanolamine (DOPE), 1,2-dioleoyl-3-trimethylammonium-propane (DOTAP), cholesterol (CHOL) and carboxyfluorescein-labeled DOPE (1,2-dioleoyl-sn-glycero-3phosphoethanolamine-N-(carboxyfluorescein) (ammonium salt)) were purchased from Avanti Polar Lipids (Alabaster, AL, USA). FA-PEG $2000-D S P E$ was purchased from Ponsure Biotechnology (Shanghai, China, Lot No. B06259). $\mathrm{H}^{1}$-NMR and MALDI-TOF were performed to identify its chemical structure and molecular weight (Fig. S1 and S2). Lyso-Tracker Red was purchased from Beyotime Biotechnology (Nantong, China). Rhodamine-labeling for pDNA was performed using IT $^{\circledR}$ Rhodamine labeling kit (Mirus, Madison, WI, USA) according to the manufacturer's protocol. Polyethylenimine (PEI $25 \mathrm{~K}$ ), filipin, amiloride, and chlorpromazine were obtained from Sigma-Aldrich (St. Louis, MO, USA). Hela and HEK 293 cells (American Type Culture Collection, Rockville, MD, USA) were cultured in RPMI 1640. The media were supplemented with $10 \% \mathrm{FBS}, 100 \mathrm{U} / \mathrm{ml}$ penicillin/streptomycin. Cells were grown in a humidified incubator at $37{ }^{\circ} \mathrm{C} / 5 \% \mathrm{CO}_{2}$. The plasmid DNA templates were the SB transposon system, made of a transposon encoding EGFP ( $\mathrm{pT}_{2} \mathrm{EGFP}$ ) and a plasmid encoding transposase (SB11), which was reported in our earlier work [7]. DNA products of PCR amplification contained a CMV promotor as well as the transposon and transposase gene. DNA primers and TET labeled primers were purchased from Sangon Biotech (Shanghai). The primer sequence is listed as follow: for $\mathrm{pT}_{2} \mathrm{EGFP}$, primer 1: 5'-gcgcagttgaagtcggaagt-3', primer 

5 '-ccegcggccaattctagtat-3'.

\subsection{Preparation of protocells and DNA amplification}

Protocells were prepared by thin film hydration following a former report [15]. Briefly, DPPC, DOPE and cholesterol (5:4:1, molar ratio) were dissolved in chloroform. The organic solvent was evaporated and the lipids were dried to form a thin film by $\mathrm{N}_{2}$. For FA modification, 5\% FA-PEG 2000 -DSPE of total lipid was added in chloroform to make the thin film for protocell preparation. To integrate DEX into the protocells, DEX, instead of cholesterol, was added in chloroform with other lipid to prepare the thin film $[18,19]$. Then, the PCR solution, containing the transposon/transposase DNA template $(0.1$ $\mathrm{ng} / \mu \mathrm{L})$, primers $(0.8 \mu \mathrm{M})$, dNTPs $(0.2 \mathrm{mM}$ each) and polymerase $(0.025 \mathrm{U} / \mu \mathrm{l})$, was added and the thin film was hydrated and dispersed by vortex mixing. The total concentration of lipid was $0.3 \mathrm{mM}$ for the final sample. The prepared protocells were treated with 20 PCR thermal cycles (Table 1). DOTAP liposomes (with a molar ratio of DOTAP:DOPE of 1:1, and a total concentration of lipid of $0.3 \mathrm{mM}$ ) were prepared by the thin film method as a control sample

Table 1. PCR conditions for protocell preparation.

\begin{tabular}{cccc}
\hline Cycle number & Denaturation & Annealing & Elongation \\
\hline 1 & $95^{\circ} \mathrm{C}$ for $30 \mathrm{~s}$ & & \\
\hline $2 \sim 21$ & $95^{\circ} \mathrm{C}$ for $30 \mathrm{~s}$ & $55^{\circ} \mathrm{C}$ for 2 min & $72^{\circ} \mathrm{C}$ for 1 min \\
\hline 22 & & & $72^{\circ} \mathrm{C}$ for $1 \mathrm{~min}$ \\
\hline
\end{tabular}

\subsection{Characterization of protocells}

The size and zeta-potential values of protocells were determined by dynamic light scattering (DLS) on a zetasizer Nano ZS (Malvern, UK), including protocells before and after PCR amplification and FA-protocell loading with DEX. The morphology of protocells was observed using transmission electron microscopy (TEM, Tecnai G2 F30, FEI, USA). To prepare TEM samples, one drop of protocells was placed on a copper grid and stained with $3 \%$ phosphotungstic acid for $30 \mathrm{~s}$. The grid was allowed to dry for 20 min and then examined with the electron microscope. 
To detect the amplified linear DNA of PCR product, the protocells were treated with DNase Ifor $5 \mathrm{~min}$ at $37{ }^{\circ} \mathrm{C}$ Then, $20 \mu \mathrm{L}$ mixtures of Tris-saturated phenol and chloroform $(1: 1, V: \mathrm{V})$ were added into $10 \mu \mathrm{L}$ sample of protocells to remove the lipids and enzymes. The liquid supernatant containing PCR product of the protocell solution was electrophoresed on a $0.8 \%(\mathrm{w} / \mathrm{v})$ agarose gel in TAE buffer at $90 \mathrm{~V}$ for $40 \mathrm{~min}$, and analyzed on a UV illuminator to show the location of the DNA.

\subsection{Transfection efficiency of protocells}

For the transfection experiments, the cells were seeded in the 24-well plate at a density of $1 \times 10^{5}$ cells/well and incubated overnight. The protocells were added into the cells at different lipids concentrations containing PCR products of transposons $\mathrm{pT}_{2} \mathrm{EGFP}$ and transposase SB11. After incubated for $4 \mathrm{~h}$ at $37^{\circ} \mathrm{C}$, the transfection medium was replaced with a fresh culture medium and the cells were further incubated for $24 \mathrm{~h}$. The transfected cells were digested by trypsin and fixed in a 4\% paraformaldehyde for $30 \mathrm{~min}$ to prepare single-cell suspensions. The percentage of cells positive for GFP was determined by flow cytometry (Becton-Dickinson Biosciences, USA). In control experiments, DOTAP liposomes at different lipid concentrations or PEI 25K were mixed with the SB transposon system $\left(1 \mu \mathrm{g} \mathrm{pT}{ }_{2}\right.$ EGFP and $\left.1 \mu \mathrm{g} \mathrm{SB100}\right)$ and incubated for $15 \mathrm{~min}$ at room temperature for further use.

\subsection{Dexamethasone loaded protocells for nuclear translocation}

DEX, a well-known synthetic steroidal glucocorticoid, shows structural resemblance with cholesterol (Chol) and hence afforded stable formulation in the presence of lipid [18, 20]. Thus, DEX was directly incorporated alongside the regular phospholipid in the lipidassociated gene delivery formulation.

For fluorescence imaging, the primer was labeled with TET and carboxyfluoresceinlabeled DOPE was used to prepare protocells. Then, PCR amplification was performed to get double fluorescent labeled protocells. The Hela cells were grown on glass cover slips in 6-well plates and incubated at $37{ }^{\circ} \mathrm{C}$ for a day. The cells were transfected by labeled protocells, and incubated for $4 \mathrm{~h}$ at $37^{\circ} \mathrm{C}$. The transfection medium was then replaced with a fresh medium. After $8 \mathrm{~h}$, the cells were fixed with $4 \%$ paraformaldehyde for 20 
min. To stain the cell nuclei, the cells were incubated with Hoechest 33342 for $15 \mathrm{~min}$ at room temperature, and then the cover slips were mounted on glass slides. The fluorescence was observed with a confocal laser scanning microscope (Leica TCS SP8, Germany).

\subsection{Identification of cellular uptake pathways}

In order to determine the intracellular location of protocells, protocells were labeled with carboxyfluorescein DOPE and incubated with Hela cells for $1 \mathrm{~h}$. Then, the cells were washed with PBS and $100 \mathrm{nM}$ of Lyso-Tracker Red was added and incubated for 30 min. After the staining solution was removed, the cells were rinsed twice with PBS (pH 7.4) and fixed with $4 \%$ paraformaldehyde for 20 min. Heochest 33342 was applied to stain the nuclei for another $15 \mathrm{~min}$. Then the cells were rinsed with cold PBS and observed by Confocal Microscope (Zeiss, USA).

For quantifying intracellular transport pathways of protocells, different endocytosis inhibitors were used, including chlorpromazine $(30 \mathrm{mM})$, a clathrin-mediated endocytosis inhibitor, filipin (15 mM), a caveolae-mediated endocytosis inhibitor, and amiloride (50 $\mathrm{mM})$, a macropinocytosis inhibitor [21, 22]. Each endocytosis inhibitor was added to the culture medium and incubated with cells for $30 \mathrm{~min}$. Then, the protocells labeled with carboxyfluorescein DOPE were used to transfect cells for $4 \mathrm{~h}$. Cells were trypsinized and suspended, and the fluorescence intensity of the resultant supernatant was measured by flow cytometry. Transfected cells without treatment of endocytosis inhibitors were used as a positive standard.

\subsection{Cytotoxicity assay}

Cytotoxicity of protocell and DOTAP liposome were evaluated by MTT assay. Briefly, cells were seeded in 96-well plates at 5000 cells per well. One day later, the cells were treated with protocells and DOTAP liposomes at different lipids concentrations. The cells were incubated for $36 \mathrm{~h}$ at $37^{\circ} \mathrm{C}$. The medium was replaced with $100 \mu \mathrm{L}$ of fresh medium without serum and $20 \mu \mathrm{L}$ of MTT $(5 \mathrm{mg} / \mathrm{mL})$ solution, and incubated for an additional $4 \mathrm{~h}$. Subsequently, the medium was removed and $100 \mu \mathrm{L}$ of DMSO was added. 
The absorbance at $490 \mathrm{~nm}$ was measured by using a microplate reader. PEI $25 \mathrm{~K}$ and DOTAP liposome were used as the control.

\subsection{In vivo study}

To generate tumor models, six-week old female BALB/c nude mice were subcutaneously injected in the subaxillary region with Hela cells $\left(1 \times 10^{7}\right.$ cells $)$ suspended in $200 \mu \mathrm{L}$ of PBS. Experiments were carried out when tumors reached approximately 200-300 $\mathrm{mm}^{3}$. Subsequently the unmodified protocells and FA-protocells were intravenously injected into the mice separately. PBS solution was injected instead in a control group. The doses of the unmodified protocells and FA-protocells per mouse were $50 \mu \mathrm{g}$ DNA oligomer of $\mathrm{pT}_{2} \mathrm{EGFP}$ and SB100, respectively. Twenty four hours after injection of the transfection reagents, the mice were sacrificed. Then the tumor tissue as well as the control tissues (heart, liver, spleen, lung, and kidney) were excised and washed with PBS. To perform cryofixation, the tissues were immersed in Tissue-Tek OCT embedding medium (Sakura, Torrance, CA, USA) followed by freezing at $-80{ }^{\circ} \mathrm{C}$. Then the resultant frozen sections $(5 \mu \mathrm{m}$ thick) were prepared with Cryostat Microtome (CM 1900; Leica, Wetzlar, Germany) following a published method [23]. The tissue sections were placed on the slides and imaged by a fluorescence microscope to detect EGFP fluorescence. In order to quantify the number of EGFP positive cells in different tissues, single-cell suspensions were also derived from the tissues by mechanical disruption. The cells from different tissues were washed twice and fixed in $4 \%$ paraformaldehyde for $30 \mathrm{~min}$ at room temperature, and then resuspended in PBS. The percentage of EGFP positive cells was measured by flow cytometry and 10,000 cells were analyzed in each sample [24]. The protocol of the in vivo study was approved by the Institutional Animal Care and Use Committee (IACUC) of Dalian University of Technology.

\subsection{Statistical analysis \\ Data are given as mean \pm standard deviation. Statistical significance between treatments was tested by one-way analysis of variance analysis (ANOVA). The p-value for significance was set at 0.05 .}




\section{Result and Discussion}

\subsection{Preparation and characterization of protocells}

To prepare the protocells, the transposons DNA template and PCR components (primers, dNTPs and polymerase) were encapsulated into neutral lipid-based protocells composed of DPPC, DOPE and Cholesterol/DEX, immediately followed by 20 PCR cycles (Table 1). For surface modification, FA-PEG 2000 -DSPE was added to prepare the protocells functionalized with cell targeting FA (Fig. 1). After DNA amplification, the sizes of the protocells were detected by DLS, showing no significant difference in the size of the protocells before $(226.8 \pm 6.1 \mathrm{~nm})$ and after $(223.1 \pm 4.9 \mathrm{~nm})$ PCR cycles. TEM imaging confirmed that the size of the protocells was about $200 \mathrm{~nm}$ and not affected by DNA amplification (Fig. 2), indicating the thermal stability of the protocells during the high temperature PCR amplification. There are no significant structural damages and no formation of aggregates following the temperature cycles. The size of FA-protocells (Table S1), namely, the protocells modified with FA-PEG $2000-\mathrm{DSPE}$, is $230.0 \pm 4.4 \mathrm{~nm}$, which is similar to the unmodified ones, suggesting that FA-PEG $2000^{-}$ DSPE modification didn't change the size of protocells as well. The surface charge of the protocells was slightly negative before $(-2.13 \pm 0.19 \mathrm{mV})$ and after $(-2.15 \pm 0.19 \mathrm{mV})$ PCR amplification. After modified by FA-PEG 2000 -DSPE, the protocells exhibited an almost neutral surface charge $(-1.35 \pm 0.07 \mathrm{mV})$. Because FA-PEG ${ }_{2000}$-DSPE is a neutral compound with a long chain, modification of the protocells by FA-PEG ${ }_{2000}-\mathrm{DSPE}_{\text {made }}$ their surface potential of protocells become more neutral. 


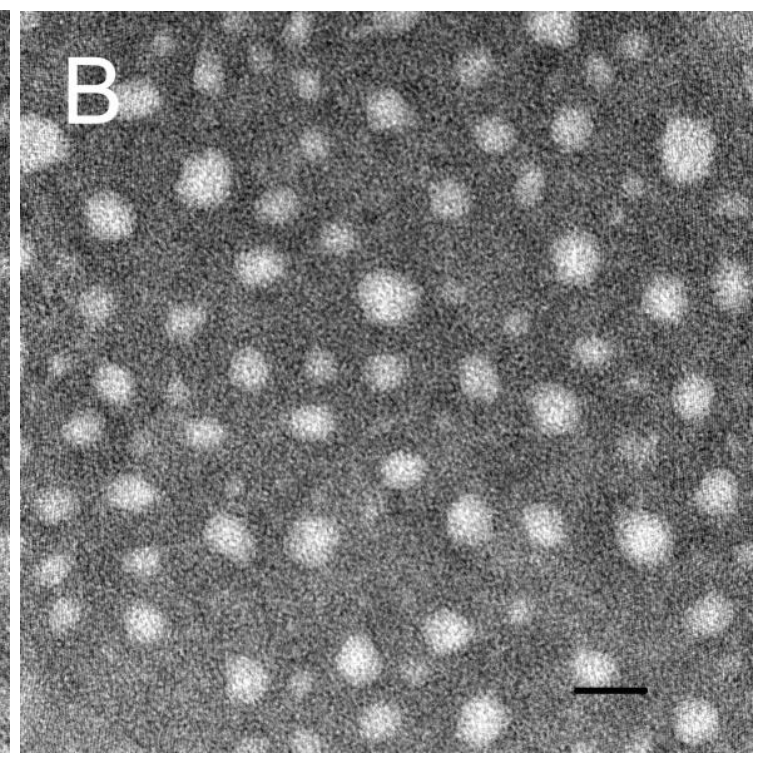

Fig. 2. Morphology of the protocells before (A) and after (B) PCR amplification as observed by TEM. The protocells were negatively stained by a $3 \%$ aqueous solution phosphotungstic acid for 30 s. Scale bar: $200 \mathrm{~nm}$.

\subsection{Amplified DNA oligomers in protocells}

To investigate the protective effect of the protocells, DNase Iwas added to digest the DNA product inside the protocells, and the remained DNA oligomers were checked by agarose gel electrophoresis. To detect the DNA products in the protocells, the lipid constituting the protocells was removed by Tris-saturated phenol-chloroform $(1: 1, \mathrm{~V}: \mathrm{V})$ after PCR amplification. The DNA extract solution in the supernatant was analyzed by agarose gel electrophoresis.

In this study, the PCR products in the protocells included a CMV promotor, a transposon ( $\left.\mathrm{pT}_{2} \mathrm{EGFP}\right)$ and/or a transposase gene (SB100). The amplified DNA fragment is expected to be $1867 \mathrm{bp}$ long for SB100 and $2136 \mathrm{bp}$ long for $\mathrm{pT}_{2} \mathrm{EGFP}$, respectively, which is confirmed by agarose gel images (Fig. 3A-a and 3B-a). After digested by DNase I, the DNA products amplified inside the protocells still remained intact (Fig. 3. $\mathrm{A}, \mathrm{b} ; \mathrm{B}, \mathrm{b})$, while the DNA products derived from the conventional PCR amplification were degraded completely (Fig. 3. A,c; B,c). These results indicated that the protocells could efficiently incorporate a high concentration of DNA oligomers into a neutral lipid formulation by means of PCR amplification, implying that much more gene materials 
could be delivered for better cell uptake. Furthermore, under the protection of the protocells, the DNA products became more stable and were not affected by biological macromolecule materials (e.g., DNase) outside the protocells. All of these features will favor better transgenic expression. In a control experiment, we used a cationic lipid, DOTAP, to prepare the protocells. But no DNA oligomers could be produced after PCR (data not shown), further confirming that cationic protocells are not appropriate for performing PCR amplification [15].

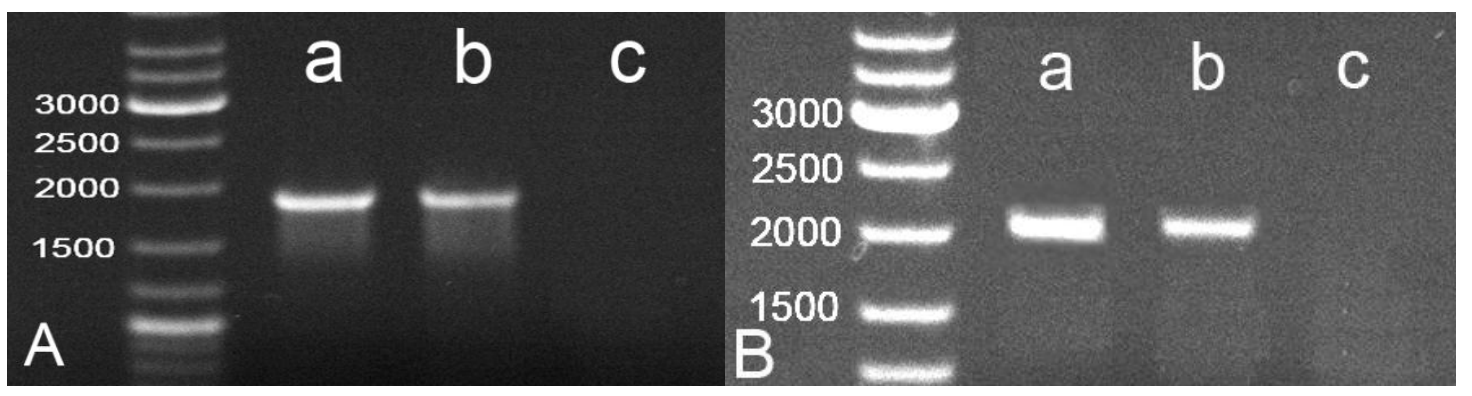

Fig. 3. Agarose gel image of DNA product of $\mathrm{SB} 11$ (A) and $\mathrm{pT}_{2} \mathrm{EGFP}(\mathrm{B})$ after PCR amplification. Lane a: Agarose gel image of amplified linear DNA after 20 PCR cycles in the protocells; Lane b: Amplified linear DNA encapsulated in the protocells after DNase I treatment. After PCR processes, DNase I was used to remove DNA remaining outside the protocells; Lane c: Amplified DNA oligomers in the conventional PCR method, followed by DNase I treatment.

\subsection{Targeted gene delivery to cancer cells by protocells}

To evaluate the transfection efficiency, the protocells containing transposon oligomers and transposase oligomers were mixed together at a mass ratio of 2:1 and added to Hela cells. The protocells were found to reach an optimal transfection efficiency $(\sim 20 \%)$ at 0.3 mM lipid, which was slightly lower than that of DOTAP liposome $(\sim 30 \%)$ but much higher than that of DPPC/DOPE liposome/DNA mixture ( 5\%) (Fig. 4A). The mixture of the DPPC/DOPE liposome and the PCR product simply wasn't able to carry DNA to the cells due to the lack of charge-charge interactions between DNA oligomers and neutral liposomes in the mixture, resulting in poor DNA loading efficiency in neutral liposomes and the subsequent low transgenic expression. The protocells containing a high concentration of DNA oligomers could deliver plenty of gene materials into the cells. 
However, because of their neutral surface, they were less likely to electrostatically interact with cells than the cationic DOTAP liposomes, leading to a lower transfection efficiency of the former than the latter.
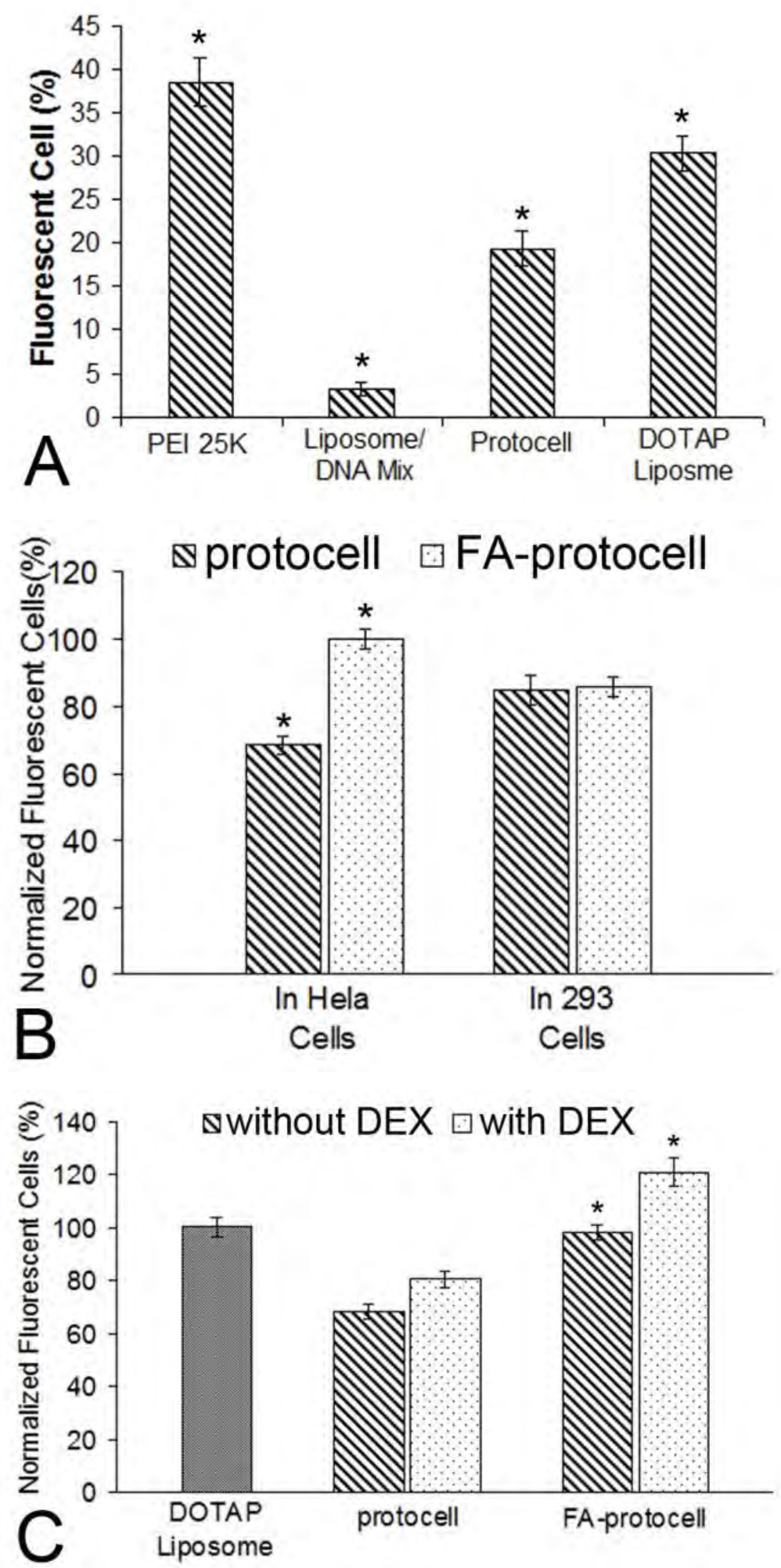
Fig. 4. In vitro transgenic expression of protocells. (A) Efficiency of transfection in Hela cells by different gene carriers, including Liposome/DNA mixture, un-modified protocells, DOTAP liposome, and PEI 25K. All formulations contain $0.3 \mathrm{mM}$ of lipids. (B) Efficiency of transfection of unmodified protocells and FA-protocells. In Hela cells, EGFP expression of FA-protocell was 1.5 times higher than that of unmodified protocells. But no obvious difference was observed in HEK 293 cells between unmodified and modified protocells. FA-protocells in Hela cells were set as a standard control and the transfection efficiency by this vector was normalized to $100 \%$. (C) The effect of integrating DEX into the protocells on the transfection efficiency in Hela cells. After loaded with DEX, transgenic expression of both unmodified protocells and FA-protocells was increased significantly. DOTAP liposome was used as a standard control and the transfection efficiency by this vector was normalized to $100 \%$.

To improve the transfection efficiency of the protocells, they were modified with FA, a ligand capable of targeting cancer cells [25-27]. After modified with $\mathrm{FA}-\mathrm{PEG}_{2000}-\mathrm{DSPE}$, the protocells exhibited a transfection efficiency in Hela cells that was 1.5 times higher than that of the unmodified protocells and also comparable to that of DOTAP liposome (Fig. 4B) [28]. To verify the cancer cell-targeting ability of the FA-modified protocells (FA-protocells), HEK 293, a type of non-cancer cells, was used as a control cell for gene transfection. As shown in Fig. 4B, no obvious difference in transfection efficiency was observed between the unmodified protocells and FA-protocells in HEK 293. These results indicated that the surface modification of the protocells with active targeting moieties can enhance the uptake of the gene carriers by cancer cells but not by noncancer cells.

To further enhance the transfection efficiency of the FA-protocells, we introduced DEX, a nucleus localization signaling molecule [17], into the protocells. It is known that DEX can dilate the nuclear pore up to $60 \mathrm{~nm}$ to facilitate the uptake of transfected DNA into the nucleus [29]. Incorporating DEX into the lipid formulation was executed in order to allow the protocells could to localize the genetic cargo inside the nucleus. As expected, for both the protocells without FA modification and FA-protocells, the transfection efficiency in Hela cells was increased significantly after loaded with DEX, suggesting 
that DEX could promote the nucleus-targeted gene delivery no matter what surface modification of the carriers had. This result also confirmed that DEX didn't act on the cell membrane but only on the nucleus. Namely, DEX could not enhance uptake by any cells to result in "off-target effect". Therefore, DEX is a relatively safe nucleus targeting ligand [20]. Moreover, the FA-protocells loaded with DEX showed even much higher transfection efficiency than the cationic DOTAP liposome (Fig. 4.C).

\subsection{Intracellular localization of protocells with DEX}

To investigate the intracellular localization of the protocells loaded with or without DEX, fluorescence-labeled primers and lipids were employed to prepare the protocells. The Hela cells were transfected with the double labeled protocells for $4 \mathrm{~h}$. Then, the cells were scanned with a confocal microscope. It was revealed that after loaded with DEX, many protocells were translocated into the nuclei (Fig. 5A). However, without DEX, no protocells were found in cell nuclei (Fig. 5B). These results clearly indicated that DEX could promote the protocells to translocate into the nucleus effectively, which would in turn result in the high transfection efficiency of the protocells.

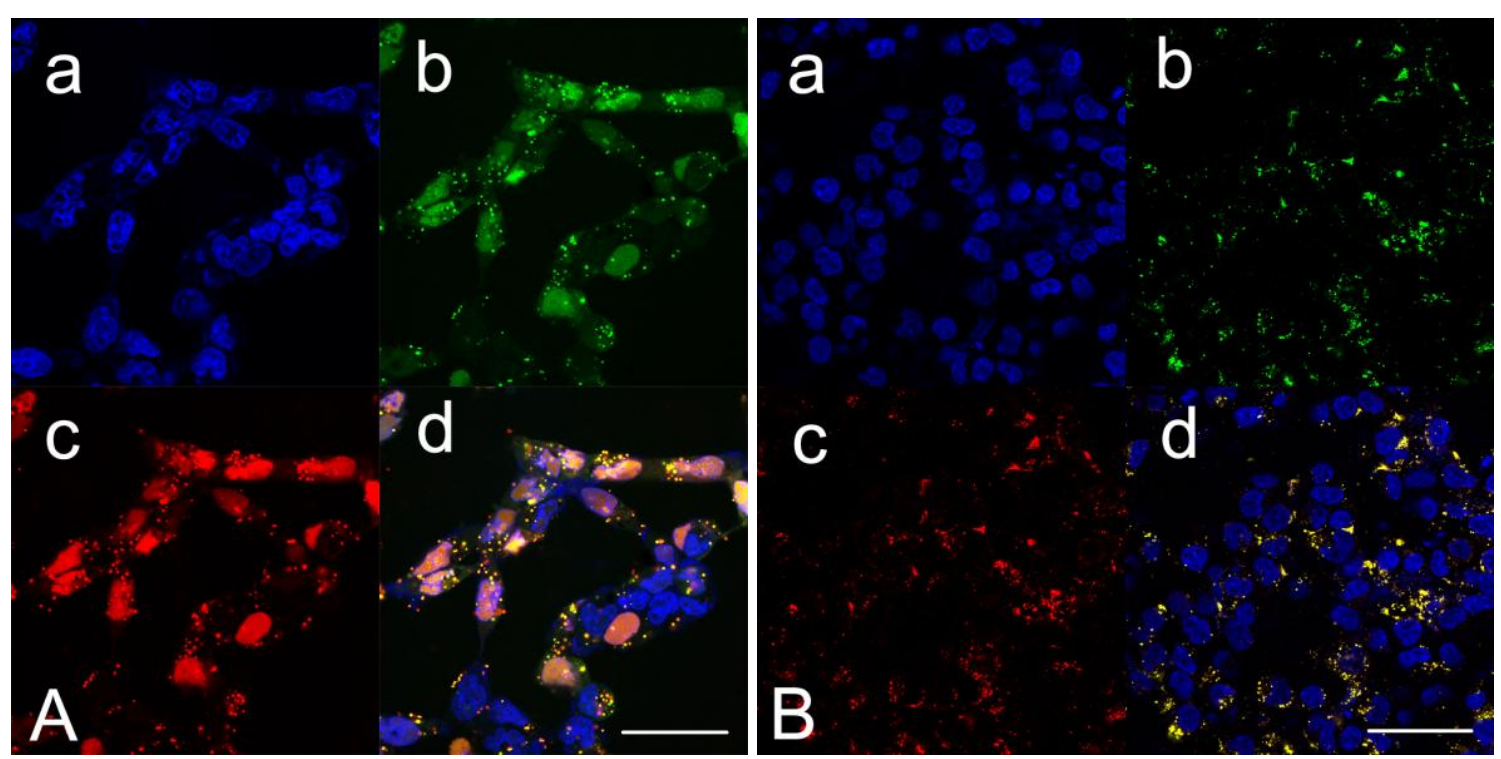

Fig. 5. Subcellular distribution of the protocells loaded with (A) and without (B) DEX visualized by confocal microscopy in Hela cells at $1 \mathrm{~h}$ after transfection. a: Hoechest 33342 labeled nuclei (blue); b: carboxyfluorescein-DOPE labeled protocells (green); c: rhodamine labeled DNA oligomers (red); d: merged images of a, b, and c. With DEX, the 
protocells and DNA oligomers could colocalize in the nuclei. However, without DEX, the protocells and DNA oligomers rarely entered the nuclei. Scale bar: $50.0 \mu \mathrm{m}$.

\subsection{Identification of intracellular transport mechanisms}

To investigate the intracellular transport mechanisms of the protocells, the intracellular amounts of the protocells were quantified in Hela cells. The protocells were labeled with carboxyfluorescein-labeled DOPE, and lysosome was labeled with Lyso-tracker Red for 30 min. Then, intracellular uptake of the protocells into the cells was studied using confocal microscopy. The protocells were observed to co-localize with lysosomes at $1 \mathrm{~h}$ after the addition of the protocells to the Hela cells (Fig. 6), indicating that that the protocells were uptaken by the cells through the mechanism of endocytosis.

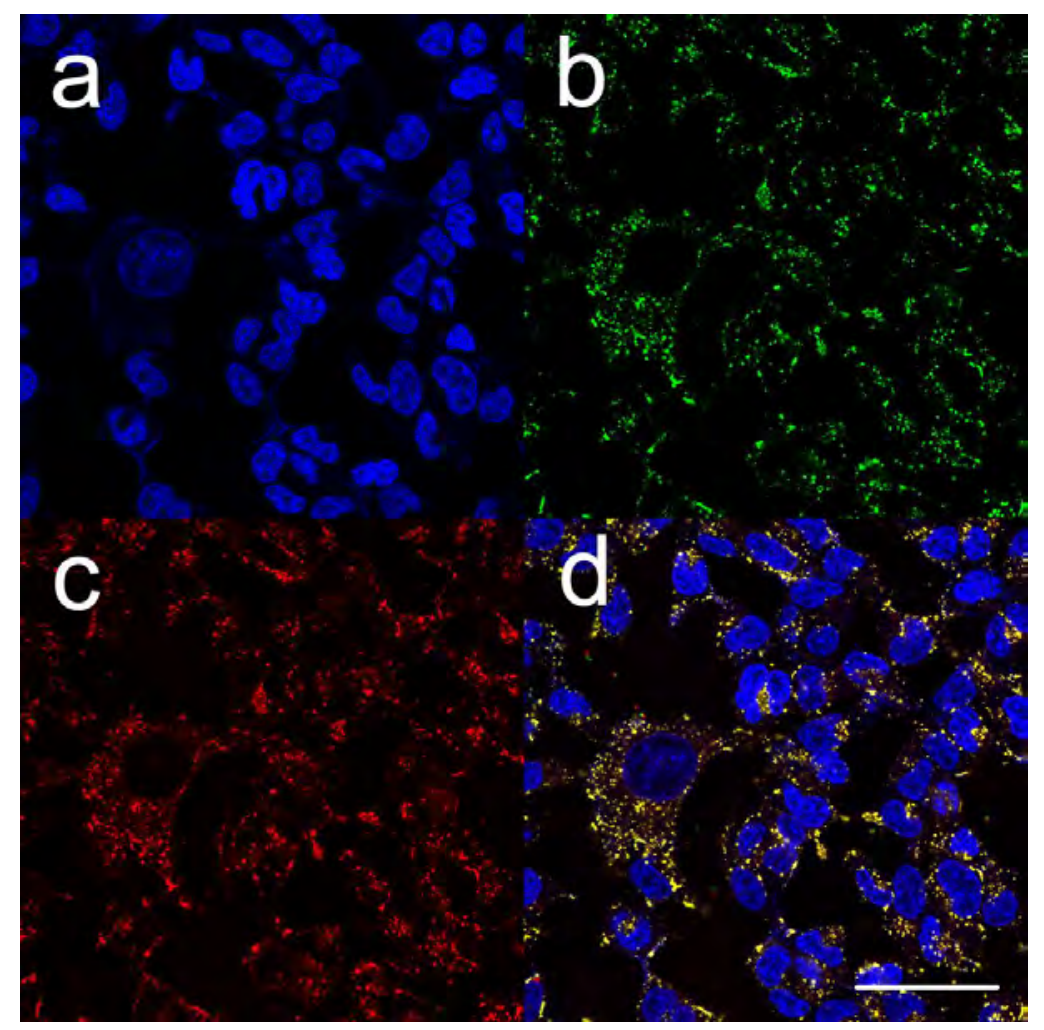

Fig. 6. Intracellular transport of the protocells characterized by confocal microscopy. (A) Confocal images showing intracellular transport at $1 \mathrm{~h}$ after the addition of the protocells to HeLa cells. The cell nuclei, protocells, lysosomes were labeled with Hoechest 33342 (a, blue), carboxyfluorescein-DOPE (b, green) and Lyso-Tracker Red (c, red), respectively. The protocells were observed to co-localize with lysosomes (d, merged image of a, b, c), 
suggesting that endocytosis is the cell uptake pathway of the protocells. Scale bars, 50 $\mu \mathrm{m}$.

To further investigate the intracellular transport mechanisms of the protocells, Hela cells were pre-treated with various endocytosis inhibitors, and then cell uptake of the protocells was detected by flow cytometry. Fig. 7 showed that no obvious caveolar dependent endocytosis was found because filipin showed no inhibition effect on the cellular uptake. Another main endocytosis pathway, macropinocytosis, was investigated utilizing amiloride. Here, macropinocytosis was not involved in the endocytosis of protocell in Hela cells (Fig. 7). But in the chlorpromazine treated group, the inhibition rates were about $25 \%$ in Hela cells, revealing the possible involvement of a clathrin dependent pathway in the endocytosis of the protocells. These observations agree with the previously published results [30-32] regarding intracellular transport mechanisms of phospholipid carriers with a similar composition. For FA-protocells, intracellular transport mechanisms were investigated in the same way. The result showed that the intracellular amounts of FA-protocells were also significantly suppressed in the presence of chlorpromazine, but not affected by other endocytosis inhibitors (Fig. 7). In general, the endocytosis pathways of the protocells with or without FA modification were similar in that they might be mediated by clathrin dependent and caveolar/macropinocytosis independent pathways. Furthermore, the results were consistent with the previous report that the internalization of lipid nanoparticles was sensitive to chlorpromazine but not to filipin or amiloride [33]. 


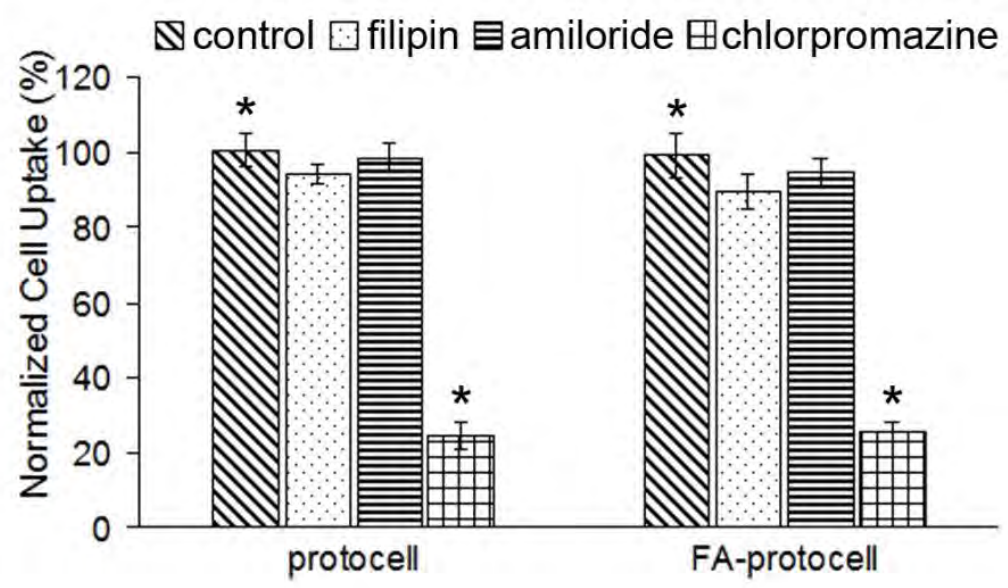

Fig. 7. The intracellular transport of the unmodified protocells or FA-protocell at $2 \mathrm{~h}$ in HeLa cells. Each endocytosis inhibitor was added to the cells at $30 \mathrm{~min}$ before the addition of the protocells. ${ }^{*} \mathrm{P}<0.05$ compared with the corresponding control group. Each value represents the mean $\pm \mathrm{SD}(\mathrm{n}=3)$.

\subsection{Cell viability of the protocells in vitro}

The cytotoxicity of the protocells was examined by means of MTT assay. As shown in Fig. 8, all gene carriers exhibited a dose-dependent cytotoxic effect. After $36 \mathrm{~h}$ incubation with cells, the cell viability of DOTAP liposome was $62 \%$ at $0.25 \mathrm{mM}$ total lipid. At 1 $\mathrm{mM}$ total lipid, the cell viability of DOTAP liposome was less $10 \%$, which showed obvious toxicity. However, the unmodified protocells and FA-protocells exhibited lower toxicity than DOTAP liposome and PEI 25K. At $1 \mathrm{mM}$ total lipid, the cell viability of both the unmodified protocells and FA-protocells was about $80 \%$, indicating almost no obvious toxicity for Hela cells. But at $1.5 \mathrm{mM}$ total lipid, the cell viability of the protocells was higher than $60 \%$, while that of the FA-protocells was only $27 \%$. It was likely that the FA-protocells with targeting capability could deliver much more DNA oligomers into Hela cells, which led to greater toxicity than the unmodified protocells. For non-cancer cell HEK 293, the cytotoxicity of the protocells with and without FA modification was similar to each other at all lipid concentrations. This result further confirmed that FA modification could improve the targeted delivery of the protocells to cancer cells but not to non-cancer cells. Overall, the cytotoxicity of the protocells with 
and without FA modification was lower than that of DOTAP liposomes, indicating that more extensive dosage range for gene transfer could be selected for the protocells.
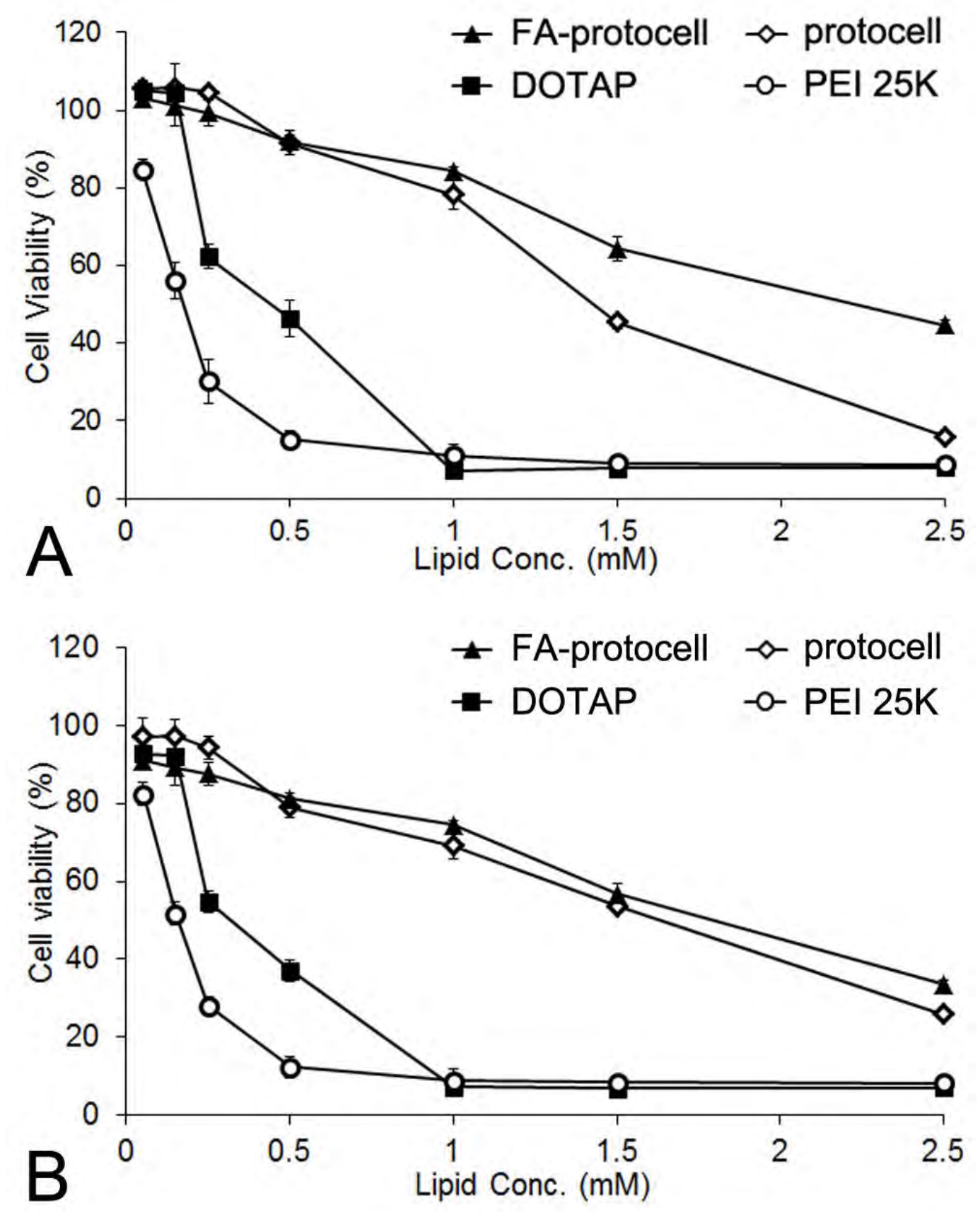

Fig. 8. MTT assay for evaluating the cytotoxicity of unmodified protocells and FAprotocells at different lipid concentrations, after incubated with Hela cells (A) and HEK 293 cells (B) for $36 \mathrm{~h}$. PEI 25K and DOTAP liposomes were used as a control. Each point represents the mean $\pm \mathrm{SD}(\mathrm{n}=3)$. 


\subsection{In vivo transfection efficiency of unmodified protocells and FA-protocells}

Transposon and transposase gene were amplified in the unmodified protocells and FAprotocells, which were injected into mice via the portal vein to investigate their transgenic activity in vivo. After $24 \mathrm{~h}$, the tumor, heart, liver, spleen, lung and kidney were harvested and their sections were made. The resultant tissue sections were then observed under fluorescence microscopy. The number of fluorescent colonies in tumor and other tissues was monitored by flow cytometry (Figure 9A). The GFP expression in different tissues was monitored by fluorescence microscopy (Figure 9B).

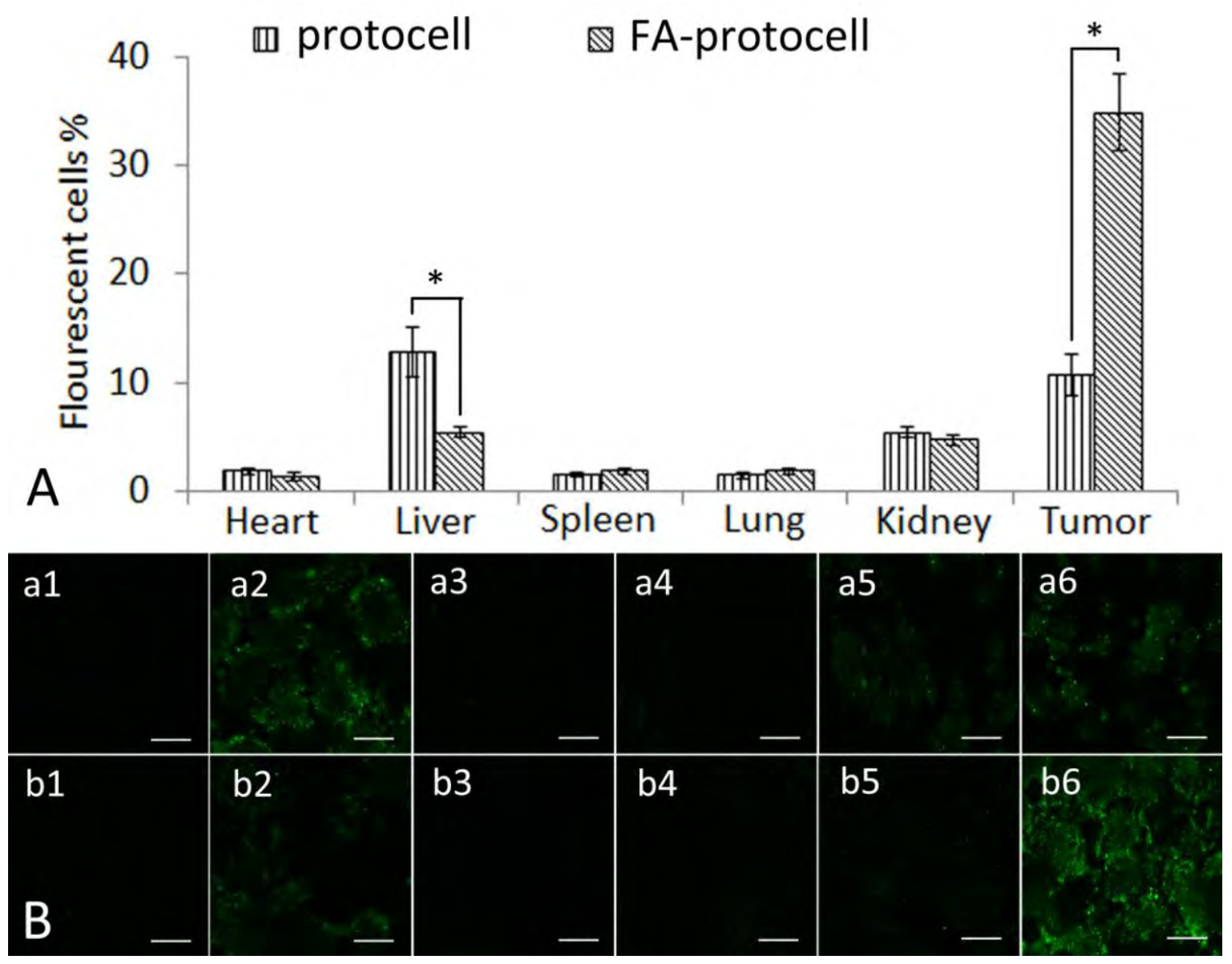

Figure 9. In vivo transfection efficiency of unmodified protocells and FA-protocells in tumor-bearing nude mice. (A) Average percentage of EGFP positive cells (in heart, liver, spleen, lung, kidney and tumor) was quantified by flow cytometry. The results are the mean \pm SD of 5 mice. (B) The qualitative evaluation of gene expression in vivo. After treated with (a) unmodified protocells and (b) FA-protocells, gene expression in different 
tissues (1: heart; 2: liver: 3: spleen; 4; lung; 5: kidney; 6: tumor) $24 \mathrm{~h}$ after portal vein administration. Frozen sections ( $5 \mathrm{~mm}$ thick) were examined by fluorescent microscopy. Scale bar $=100 \mu \mathrm{m}$.

After the mice were transfected with the unmodified protocell, the GFP positive expression efficiency was about $12.8 \%$ and $10.7 \%$ in the liver and tumor tissue, respectively (Figure 9A). After the mice were transfected with the FA-protocells, the resultant GFP positive expression efficiency increased to $34.8 \%$ in tumor but decreased to $5.4 \%$ in liver whereas the transfection efficiency in other tissues was very low (Figure 9A). Consistent with the flow cytometry assay (Figure 9A), the fluorescence image (Figure 9B) indicated that before modified with a targeting ligand, the protocells could retain in liver. This observation is reasonable because the protocells were around $200 \mathrm{~nm}$ in size, which enabled them to be easily captured by the reticuloendothelial system to target liver passively. The unmodified protocell could also reach and retain in tumor (Figure 9B), though slightly less than in liver, due to enhanced permeability and retention (EPR) effect. On the contrary, after the protocells were modified with FA, their transfection efficiency was improved significantly in tumor and decreased in liver (Figure 9A,B). Folate is a well-known ligand for solid tumor targeted delivery because folate receptor is rich on the surface of many types of cancer cells such as Hela cells. Therefore, FA could target the protocells to tumor cells and lead to the highest transgenic expression in the tumor tissues. At the same time, the PEG, used as a linker for conjugation of FA to the protocells, could avoid the capture of the protocells by the reticuloendothelial system, which in turn reduced its transgenic expression in liver. Weak EGFP expression was also observed in kidney (Figure 9B), and its reason is unclear. It should be noted that no EGFP expression was observed in PBS control group (data no shown).

\section{Conclusions}

In this study, the lipid-based protocells were used to deliver SB transposon system constituting transposon and transposase. Transposon and transposase genes could be amplified by PCR inside the protocells to achieve efficient loading and delivery. To become capable of targeting the cancer cells, the protocells were modified with FA 
ligands, resulting in a significant increase in the cell uptake in cancer cells. To further increase nuclear translocation of the genetic materials delivered into the cells by the protocells, DEX was loaded within the protocells as a nuclear localization signaling molecule. The modification of the protocells by both the FA and DEX significantly increased the transfection efficiency. Our study demonstrated that endocytosis is the cell uptake pathway of the protocells. The endocytosis of the protocells with and without FA modification was mediated by clathrin dependent and caveolar/macropinocytosis independent pathways. Both in vitro and in vivo data showed that FA-protocells could target to tumor, suggesting that the ligand-modified protocells can serve as a powerful carrier for targeted gene delivery. Because the protocells were composed of neutral lipids without cationic charges, their cytotoxicity was significantly lower than that of the traditional cationic gene carrier, such as DOTAP liposomes and PEI 25K. Hence the protocells can be used in a wider dosage range for successful gene therapy.

\section{Acknowledgment}

This study was supported by the Scientific Research Foundation for the Returned Overseas Chinese Scholars, State Education Ministry, Natural Science Foundation of China (51673168), and Zhejiang Provincial Natural Science Foundation of China (LZ16E030001). KM and CBM would also like to thank the financial support from National Institutes of Health (CA200504, CA195607, and EB021339), Department of Defense office of the Congressionally Directed Medical Research Programs (W81XWH15-1-0180), Oklahoma Center for Adult Stem Cell Research (434003), and Oklahoma Center for the Advancement of Science and Technology (HR14-160). This study was also supported by the Fundamental Research Funds for the Central Universities (NO. 5006-852010).

\section{References}

[1] Anderson WF. Human gene therapy. Nature. 1998;392:25-30.

[2] Marshall E. Clinical research. Gene therapy a suspect in leukemia-like disease. Science (New York, NY). 2002;298:34-5.

[3] Nishikawa M, Huang L. Nonviral vectors in the new millennium: delivery barriers in gene transfer. Human gene therapy. 2001;12:861-70. 
[4] Hackett PB, Ekker SC, Largaespada DA, McIvor RS. Sleeping beauty transposon-mediated gene therapy for prolonged expression. Advances in genetics. 2005;54:189-232.

[5] Sumiyoshi T, Holt NG, Hollis RP, Ge S, Cannon PM, Crooks GM, et al. Stable transgene expression in primitive human CD34+ hematopoietic stem/progenitor cells, using the Sleeping Beauty transposon system. Human gene therapy. 2009;20:1607-26.

[6] Xue X, Huang X, Nodland SE, Mates L, Ma L, Izsvak Z, et al. Stable gene transfer and expression in cord blood-derived CD34+ hematopoietic stem and progenitor cells by a hyperactive Sleeping Beauty transposon system. Blood. 2009;114:1319-30.

[7] Ma K, Wang DD, Lin YY, Wang JL, Petrenko V, Mao CB. Synergetic Targeted Delivery of SleepingBeauty Transposon System to Mesenchymal Stem Cells Using LPD Nanoparticles Modified with a PhageDisplayed Targeting Peptide. Adv Funct Mater. 2013;23:1172-81.

[8] Koo H, Jin GW, Kang H, Lee Y, Nam K, Zhe Bai C, et al. Biodegradable branched poly(ethylenimine sulfide) for gene delivery. Biomaterials. 2010;31:988-97.

[9] Hunter AC. Molecular hurdles in polyfectin design and mechanistic background to polycation induced cytotoxicity. Advanced drug delivery reviews. 2006;58:1523-31.

[10] Mann S. The Origins of Life: Old Problems, New Chemistries. Angewandte Chemie International Edition. 2013;52:155-62.

[11] Kurihara K, Tamura M, Shohda K, Toyota T, Suzuki K, Sugawara T. Self-reproduction of supramolecular giant vesicles combined with the amplification of encapsulated DNA. Nature chemistry. 2011;3:775-81.

[12] Mansy SS, Schrum JP, Krishnamurthy M, Tobe S, Treco DA, Szostak JW. Template-directed synthesis of a genetic polymer in a model protocell. Nature. 2008;454:122-5.

[13] Schroeder A, Goldberg MS, Kastrup C, Wang Y, Jiang S, Joseph BJ, et al. Remotely activated proteinproducing nanoparticles. Nano letters. 2012;12:2685-9.

[14] Oberholzer T, Albrizio M, Luisi PL. Polymerase chain reaction in liposomes. Chemistry \& biology. 1995;2:677-82.

[15] Lee S, Koo H, Na JH, Lee KE, Jeong SY, Choi K, et al. DNA amplification in neutral liposomes for safe and efficient gene delivery. ACS nano. 2014;8:4257-67.

[16] Slastnikova TA, Rosenkranz AA, Zalutsky MR, Sobolev AS. Modular nanotransporters for targeted intracellular delivery of drugs: folate receptors as potential targets. Current pharmaceutical design. 2015;21:1227-38.

[17] Rebuffat A, Bernasconi A, Ceppi M, Wehrli H, Verca SB, Ibrahim M, et al. Selective enhancement of gene transfer by steroid-mediated gene delivery. Nature biotechnology. 2001;19:1155-61.

[18] Ahmad A, Mondal SK, Mukhopadhyay D, Banerjee R, Alkharfy KM. Development of Liposomal Formulation for Delivering Anticancer Drug to Breast Cancer Stem-Cell-Like Cells and its Pharmacokinetics in an Animal Model. Molecular pharmaceutics. 2016;13:1081-8.

[19] Mukherjee A, Narayan KP, Pal K, Kumar JM, Rangaraj N, Kalivendi SV, et al. Selective cancer targeting via aberrant behavior of cancer cell-associated glucocorticoid receptor. Molecular therapy : the journal of the American Society of Gene Therapy. 2009;17:623-31.

[20] Xiong L, Du X, Kleitz F, Qiao SZ. Cancer-Cell-Specific Nuclear-Targeted Drug Delivery by DualLigand-Modified Mesoporous Silica Nanoparticles. Small (Weinheim an der Bergstrasse, Germany). 2015;11:5919-26.

[21] Un K, Sakai-Kato K, Oshima Y, Kawanishi T, Okuda H. Intracellular trafficking mechanism, from intracellular uptake to extracellular efflux, for phospholipid/cholesterol liposomes. Biomaterials. 2012;33:8131-41.

[22] Zhang Q, Tang J, Fu L, Ran R, Liu Y, Yuan M, et al. A pH-responsive alpha-helical cell penetrating peptide-mediated liposomal delivery system. Biomaterials. 2013;34:7980-93.

[23] Ma K, Hu M, Xie M, Shen H, Qiu L, Fan W, et al. Investigation of polyethylenimine-graftedtriamcinolone acetonide as nucleus-targeting gene delivery systems. The journal of gene medicine. 2010;12:669-80.

[24] Arote R, Kim TH, Kim YK, Hwang SK, Jiang HL, Song HH, et al. A biodegradable poly(ester amine) based on polycaprolactone and polyethylenimine as a gene carrier. Biomaterials. 2007;28:735-44.

[25] Wang M, Li J, Li X, Mu H, Zhang X, Shi Y, et al. Magnetically and pH dual responsive dendrosomes for tumor accumulation enhanced folate-targeted hybrid drug delivery. Journal of controlled release : official journal of the Controlled Release Society. 2016;232:161-74. 
[26] Gorle S, Ariatti M, Singh M. Novel serum-tolerant lipoplexes target the folate receptor efficiently. European journal of pharmaceutical sciences : official journal of the European Federation for Pharmaceutical Sciences. 2014;59:83-93.

[27] Li Y, Wu H, Jia M, Cui F, Lin J, Yang X, et al. Therapeutic effect of folate-targeted and PEGylated phytosomes loaded with a mitomycin C-soybean phosphatidyhlcholine complex. Molecular pharmaceutics. 2014;11:3017-26.

[28] Li Y, Lin J, Yang X, Li Y, Wu S, Huang Y, et al. Self-Assembled Nanoparticles Based on Amphiphilic Anticancer Drug-Phospholipid Complex for Targeted Drug Delivery and Intracellular DualControlled Release. ACS applied materials \& interfaces. 2015;7:17573-81.

[29] Shahin V, Albermann L, Schillers H, Kastrup L, Schafer C, Ludwig Y, et al. Steroids dilate nuclear pores imaged with atomic force microscopy. Journal of cellular physiology. 2005;202:591-601.

[30] Kerr MC, Teasdale RD. Defining Macropinocytosis. Traffic. 2009;10:364-71.

[31] Ziello JE, Huang Y, Jovin IS. Cellular endocytosis and gene delivery. Molecular medicine (Cambridge, Mass). 2010;16:222-9.

[32] Smart EJ, Anderson RG. Alterations in membrane cholesterol that affect structure and function of caveolae. Methods in enzymology. 2002;353:131-9.

[33] Wiranowska M, Colina LO, Johnson JO. Clathrin-mediated entry and cellular localization of chlorotoxin in human glioma. Cancer cell international. 2011;11:27. 


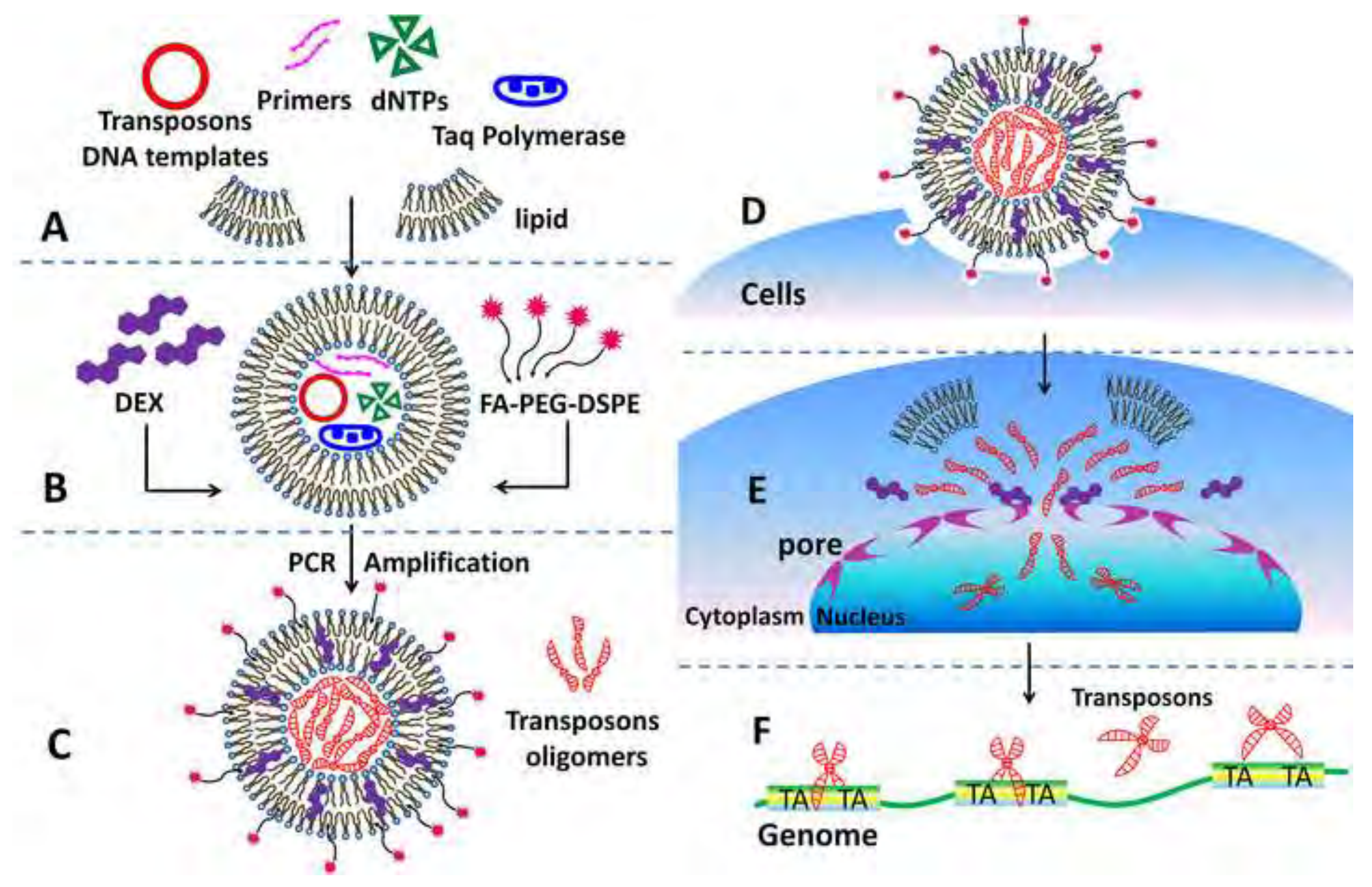

\section{Transposons}

DNA templates

c

\section{Cells}


Click here to download high resolution image
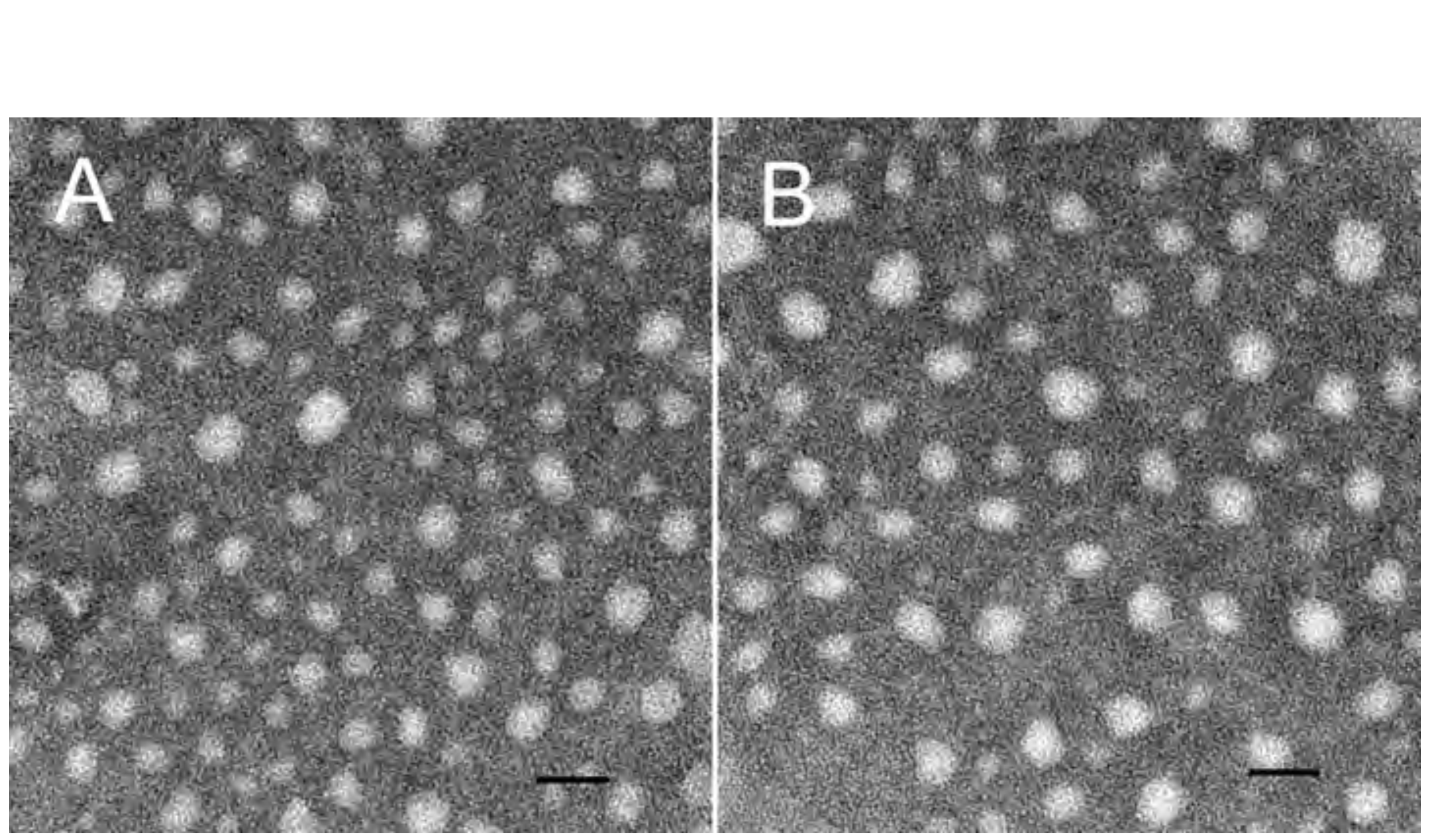
Click here to download high resolution image

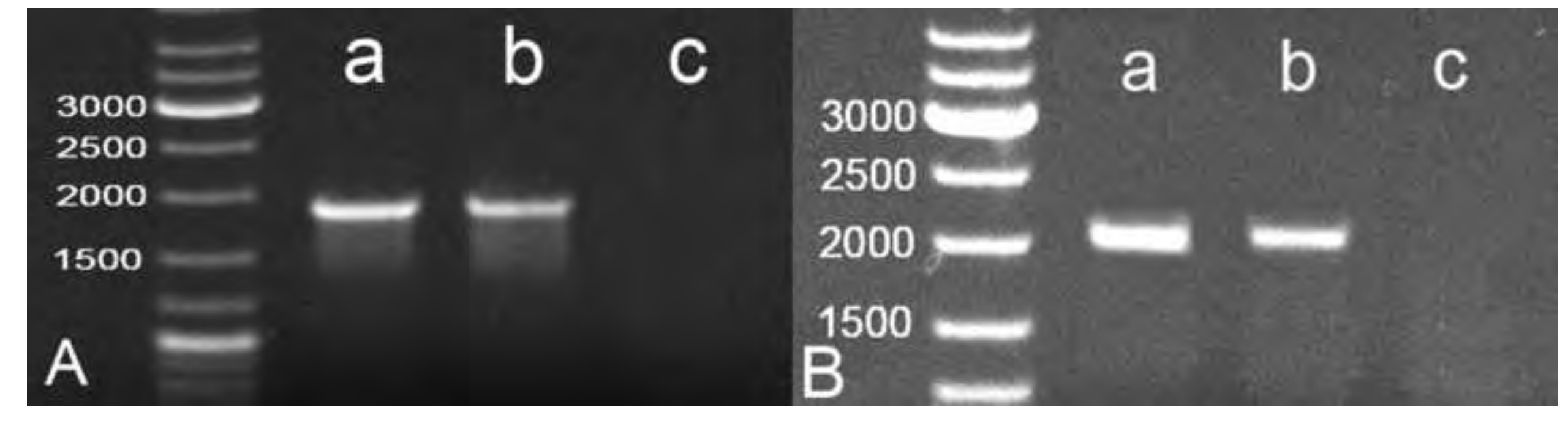



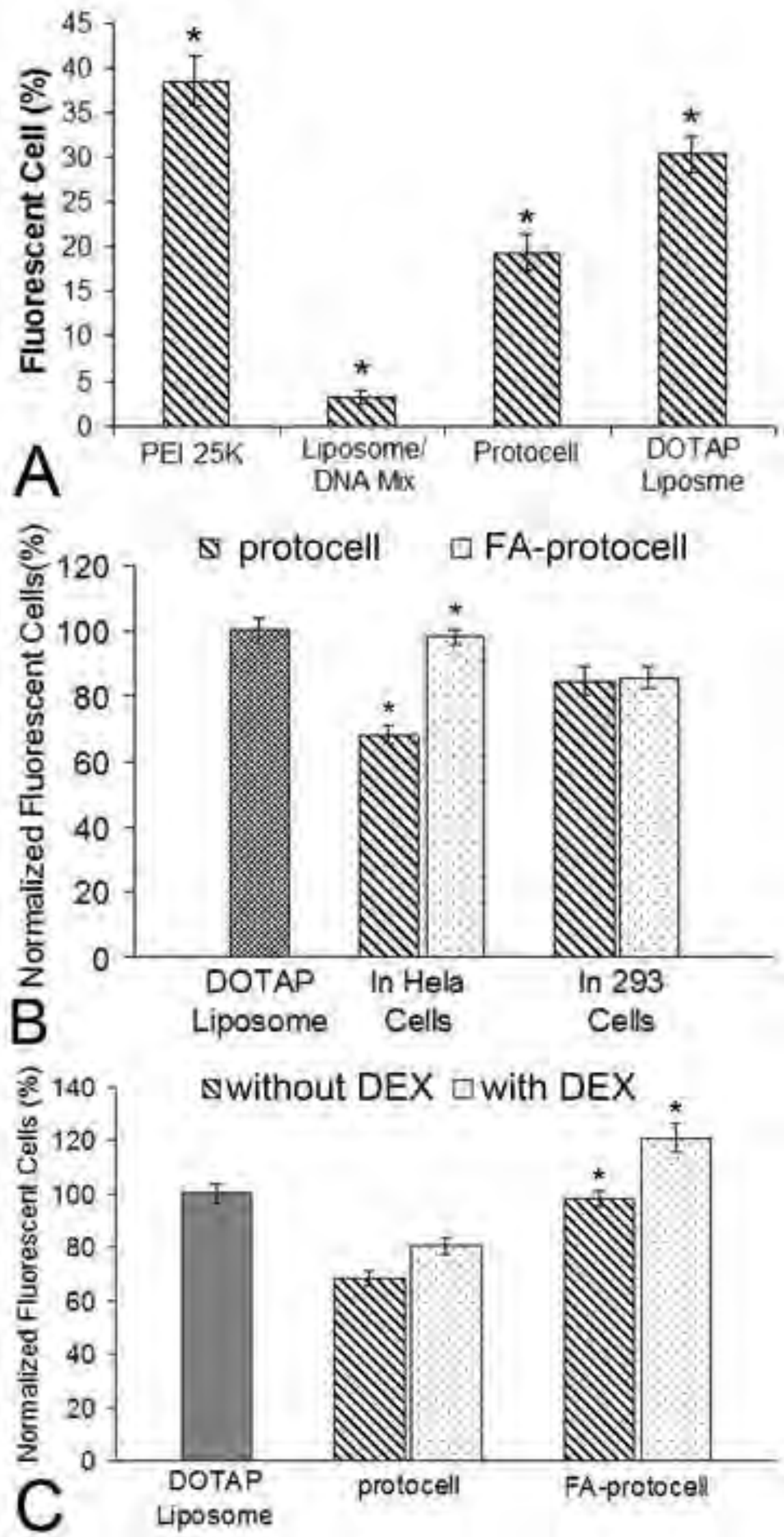


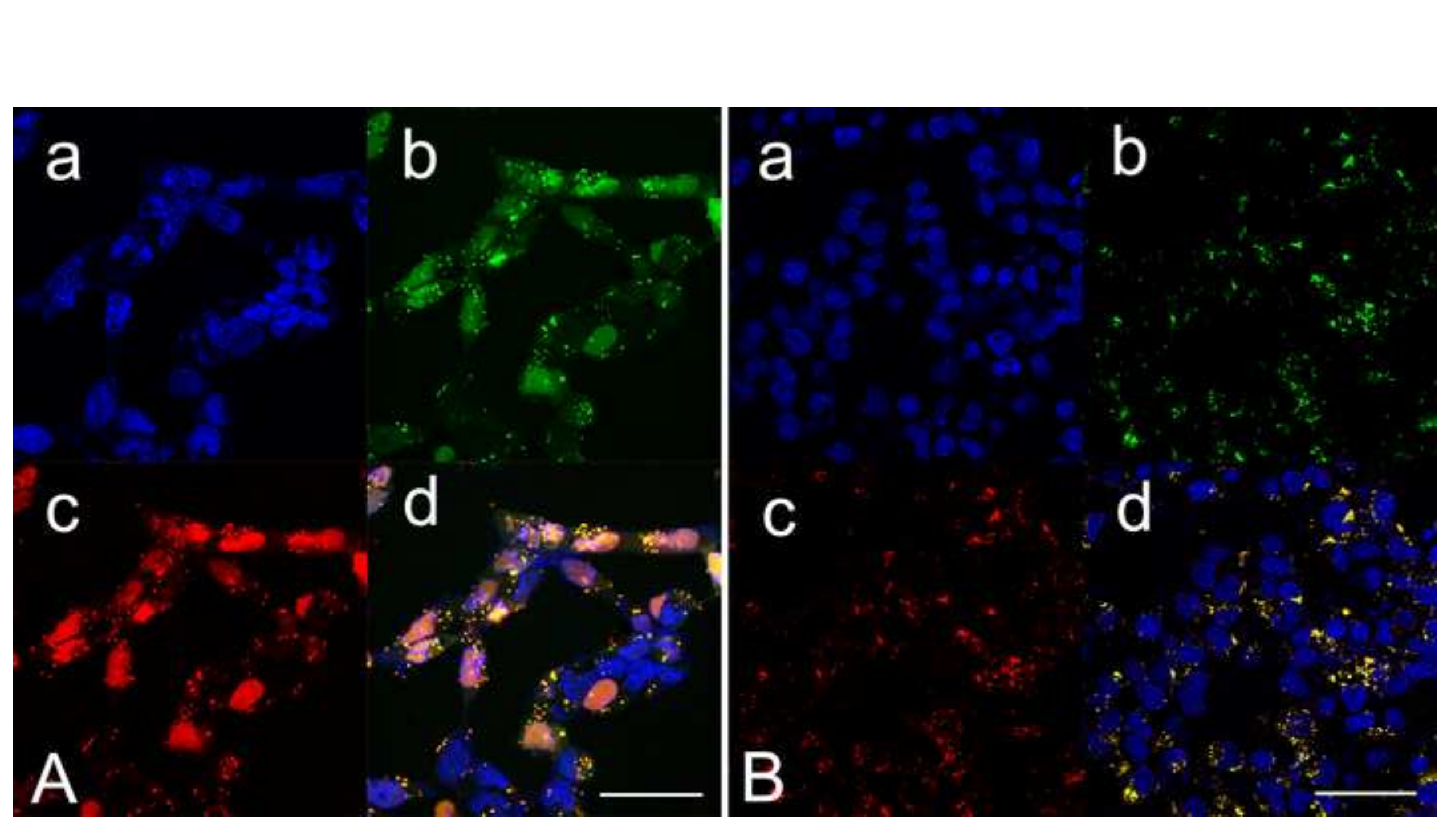


Figure

Click here to download high resolution image

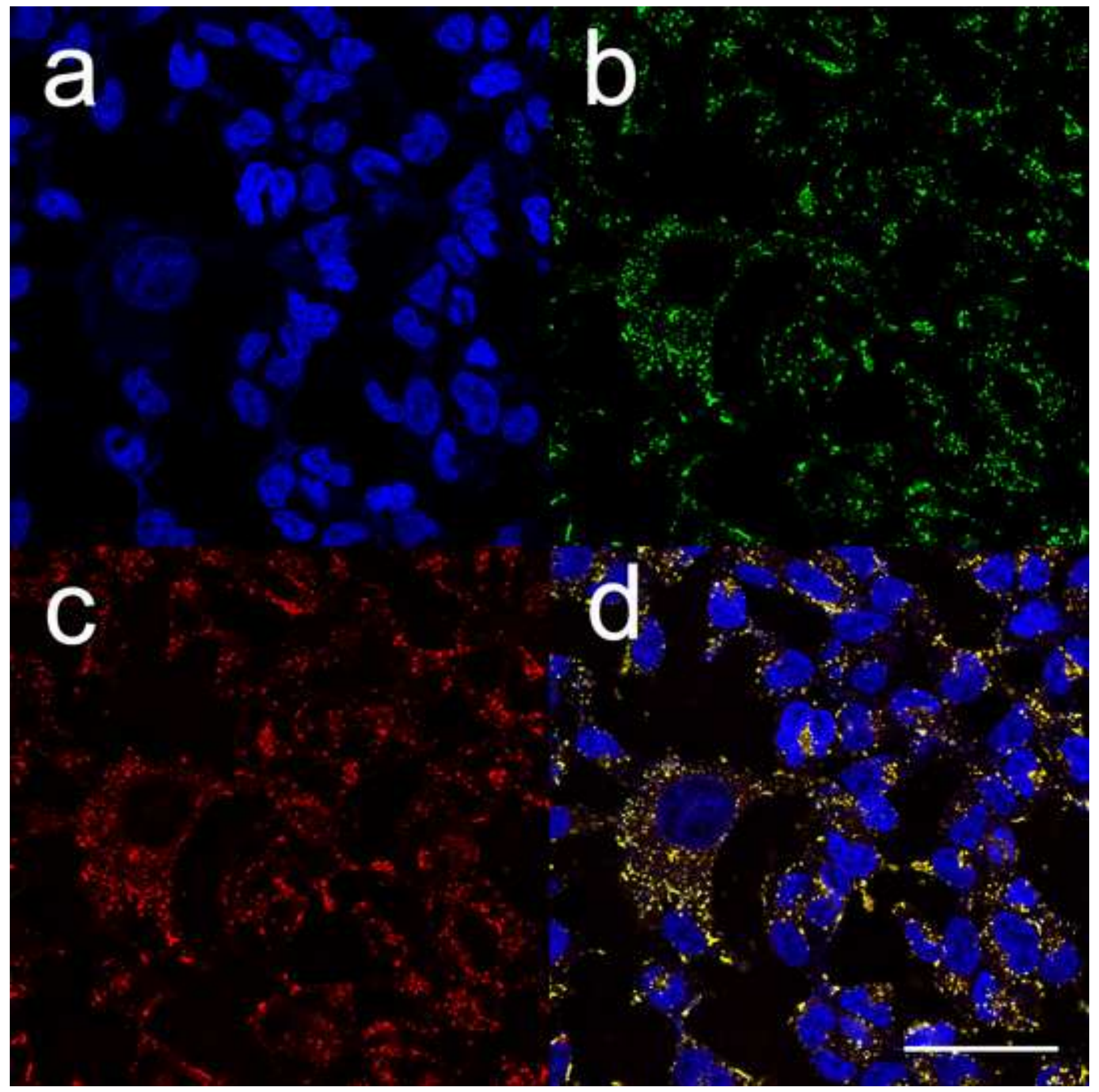


\$control $\square$ filipin 目amiloride $\boxplus$ chlorpromazine

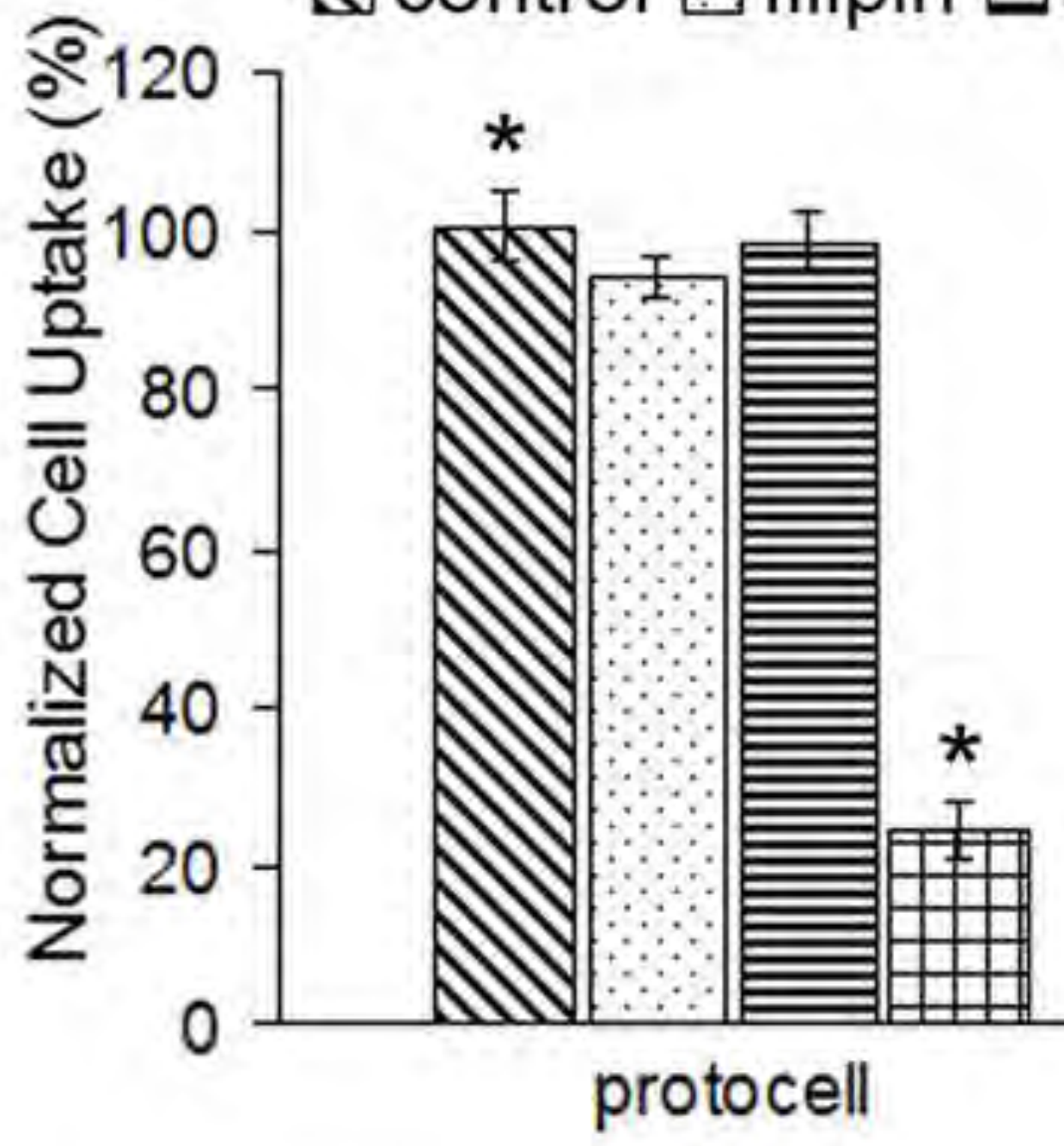

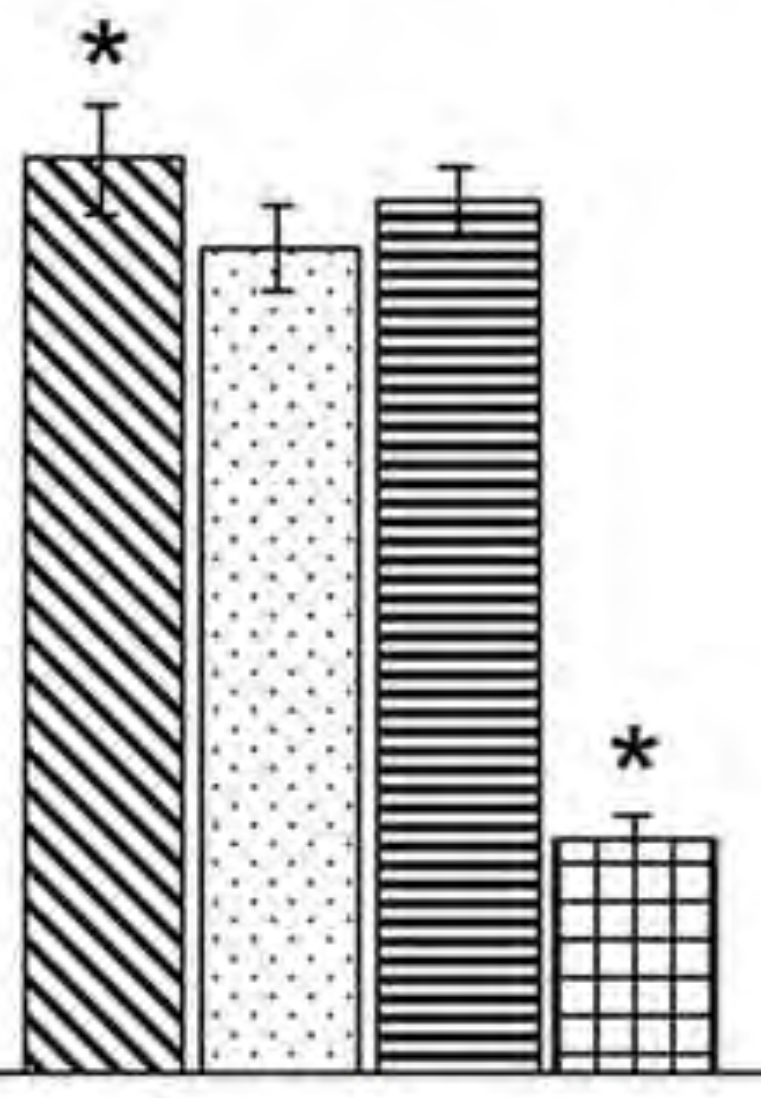

FA-protocell 
Figure

Click here to download high resolution image
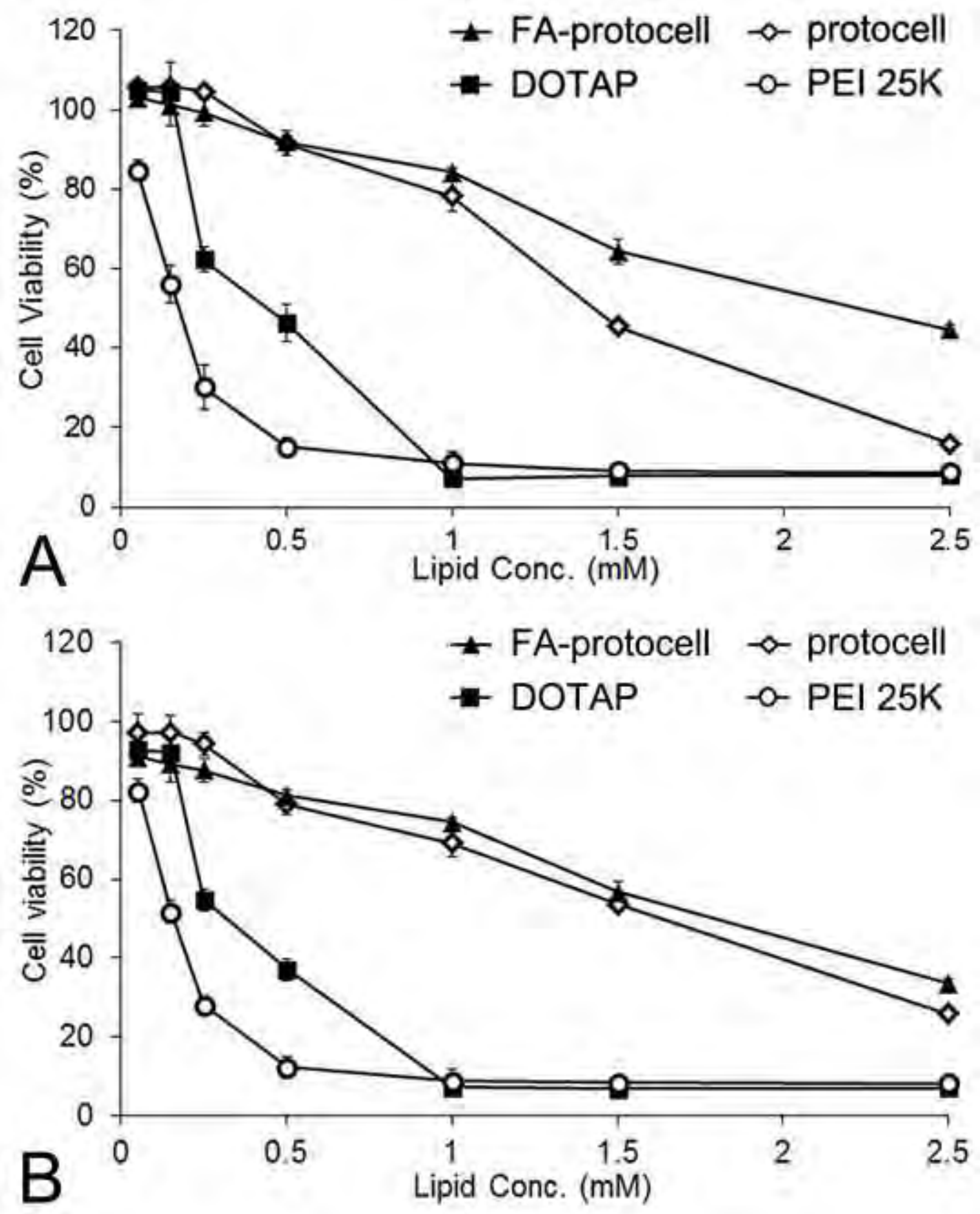
